\title{
Quantitative Comparison of Commercial CCD and Custom-Designed CMOS Camera for Biological Applications
}

\author{
Gözen Köklü Student Member, IEEE, Julien Ghaye Student Member, IEEE, René Beuchat, \\ Giovanni De Micheli Fellow, IEEE, Yusuf Leblebici Fellow, IEEE, Sandro Carrara Member, IEEE \\ École Polytechnique Fédérale de Lausanne (EPFL) - Switzerland \\ Email: name.surname@epfl.ch
}

\begin{abstract}
In biological applications and systems where even the smallest details have a meaning, CCD cameras are mostly preferred and they hold most of the market share despite their high costs. In this paper, we propose a custom-designed CMOS camera to compete with the default CCD camera of an inverted microscope for fluorescence imaging. The custom-designed camera includes a commercially available mid-performance CMOS image sensor and a Field-Programmable Gate Array (FPGA) based hardware platform (FPGA4U). The high cost CCD camera of the microscope is replaced by the custom-designed CMOS camera and the two are quantitatively compared for a specific application where an Estrogen Reception (ER) expression in breast cancer diagnostic samples that emits light at $665 \mathrm{~nm}$ has been imaged by both cameras. The gray-scale images collected by both cameras show a very similar intensity distribution. In addition, normalized white pixels after thresholding resulted in 4.96\% for CCD and $3.38 \%$ for CMOS. The results and images after thresholding show that depending on the application even a mid-performance CMOS camera can provide enough image quality when the target is localization of fluorescent stained biological details. Therefore the cost of the cameras can be drastically reduced while benefiting from the inherent advantages of CMOS devices plus adding more features and flexibility to the camera systems with FPGAs.
\end{abstract}

\section{INTRODUCTION}

The precise localization of biological details is a key aspect for many investigations on cells even at level of nucleus [1]. The most important issue is usually to get quantitative relationship between the fluorescence signals and the biological information [2], in order to distinguish closely related biological structures as inferred by fluorescence imagining [3]. These kind of investigations are usually done by using high-resolution, and usually costly CCD cameras [1], [2], [3]. Due to the high quality requirements of fluorescence imaging systems, up until recently, CMOS based cameras have not been used in this area. However, CMOS technology is a developing technology in the area of imaging and with the recent noise reduction circuits and systems, low noise, high dynamic range and high sensitivity CMOS image sensors have successfully been introduced in the high quality imaging market.

Indeed, CMOS imagers have been successfully proposed as powerful and low-cost devices for cells imaging in more recent years [4], [5] especially where the low-cost, low size and high-scale integration advantages of CMOS imagers may be unavoidable. For example, CMOS imagers have been integrated into miniature microscopes and proposed for in-vivo monitoring of biological processes on animal models [6], [7],
[8]. Contact imaging is another area of interest as described in [4] and [5] which enables imaging of samples directly on the imager without requiring any intervening optical devices. Furthermore, signal processing methods and reconstruction techniques can improve the images quality captured by CMOS devices and bridge the gap between CMOS and CCD devices.

In the following chapters, a noise reduction algorithm is introduced for Fixed Pattern Noise (FPN) reduction on data collected from CMOS sensors. Image registration and image resizing algorithms are used on the images collected by the CCD camera to achieve the same area of interest with the images collected by the CMOS camera. Later, intensity distribution histograms have been drawn from the gray-scaled images of the two cameras. Then, an auto-thresholding method is applied on the images to demonstrate that the quality of the CMOS camera is comparable with that of a CCD camera in case the required biological information is related to the localization of the morphological pattern in a biological application where in this case it is related to the detection of the ER expression.

\section{Custom-Designed CMOS CAmera}

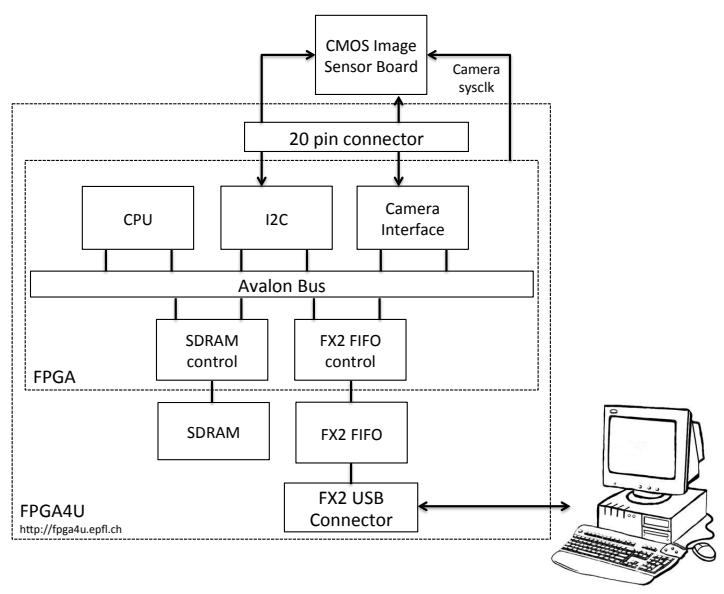

Figure 1: System Level Rep. of the custom-designed CMOS Camera

The custom-designed CMOS camera is programmed with an FPGA based hardware and software platform and includes a mid-performance black and white CMOS imager with $752 \times 480$ active pixels and 10 bits Analog to Digital Converter (ADC) resolution. On the contrary, the default CCD camera 
of the microscope includes a CCD image sensor featured with 1200x1600 unit cells and 12 bits ADC resolution. More details on the performance parameters of the two cameras can be found on Fig. 5. The custom-designed camera system mainly includes four blocks; an FPGA4u [9], a Printed Circuit Board (PCB) specifically built for the CMOS image sensor (MicronMT9V032), a case built in the workshop of EPFL including a $\mathrm{C}$ mount system and a computer connected to the FPGA4u through a USB interface in order to both program the CMOS sensor and transfer the collected images.

In Fig. 1, the system level representation of the customdesigned CMOS camera is shown. As described in this figure, the CMOS sensor is connected to the FPGA board through the 20 pin connector which has control over the $I^{2} C$ and the camera interface together. The $I^{2} C$ interface is used to configure the internal registers of the sensor and more specifically the exposure time and analog gain for this application. The captured images are stored in the SDRAM and transferred to the computer via the USB connection. The FPGA is also responsible of sending the system clock to the sensor board which is internally synchronized in the camera interface block. The camera interface inside the FPGA block consists of three main parts; a "slave part" which is an Avalon bus slave interface used to configure the camera interface, a "master part" which is an Avalon bus master interface used to store the captured mage in the SDRAM and the image acquisition part. The VHDL code of all these parts was developed and tested using the Altera Design Software. In more detail, the "Camera Synchronization" block is responsible for synchronizing the system clock coming from the FPGA board with the pixel clock of the image sensor and transfer this synchronized clock to the "Input Control" block together with the pixel data. The "Camera Controller" is responsible for the acquisition of the image and when the frame is finished, this block either captures a new frame in the video mode or waits for the next action to be taken in the snapshot mode. Then, the output signal coming from the "Camera Controller" is transmitted to the avalon bus through the "master part" for the collection of the image on the computer by the USB connector and its interfaces.

The picture of the custom-designed CMOS camera is shown in Fig. 2 which replaces the camera control unit and the CCD camera of the microscope itself. This system not only allows a low cost (one order of magnitude lower) replacement of the CCD camera but also provides a highly flexible and reprogrammable camera unit which would allow the implementation of additional functionalities and image processing algorithms directly and rapidly on board.

\section{TISSUE SAMPLE IMAGING}

\section{A. FPN Removal}

The removal of the FPN in CMOS images is very important since the intention of this research is to deal with low-light emitting samples and applications which require the images to be collected at very high analog gain and long exposure time. Since the FPN noise sources are affected by the exposure time, imager temperature and analog gain, a Master Dark Frame

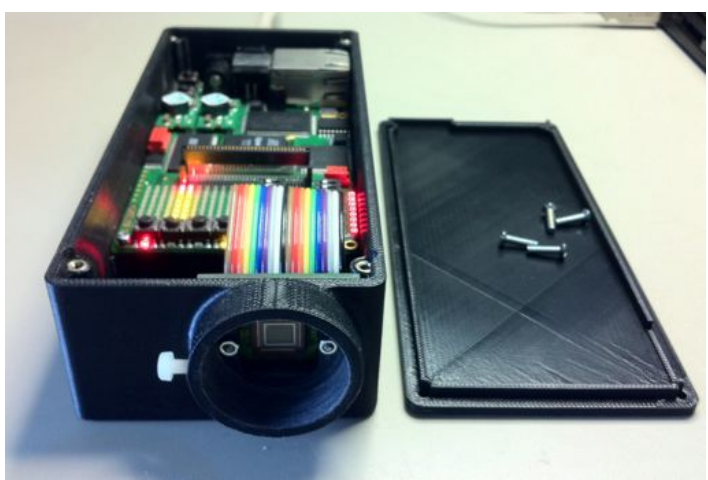

Figure 2: Overall Picture of the custom-designed Camera with Inner Blocks

(MDF) is generated by computing the median or the average frame out of a set of $N$ dark frames for each specific exposure time and analog gain (Fig. 3b). Later, the MDF is subtracted from the captured image (i.e. containing the signal) at the same exposure and gain as the MDF. The de-noised frames are computed using;

$$
F_{\text {de-noised }}=\operatorname{sat}\left(F_{\text {raw }}-M D F\right)
$$

where $\operatorname{sat}(x)$ is an operator that outputs 0 for any negative $x$ and $F_{\text {raw }}$ is the raw, noisy frame with the FPN. The sensor temperature should also be stable during this calibration process, which can be achieved by letting the system on for a few minutes before capturing the dark frames.

\section{B. Temporal Noise Removal}

Another important noise contributor in CMOS image sensors is the temporal noise which is a function of time and represents the variation of pixel outputs at constant illumination which can be modeled as a white Gaussian noise. In order to partially eliminate the temporal noise contributors, median filtering is applied on both CCD and CMOS images by which the value of an output pixel is determined by the median of the neighborhood pixels so that the outliers on the images are removed. In Fig. 3c, the corrected image of CMOS camera after FPN removal and median filtering can be seen.

\section{Image Registration and Image Resizing}

As stated earlier, the CCD and the CMOS sensors both have different pixel array sizes and pixel pitches. This causes them to image different areas of the sample, which hardens the comparison process of the sensors. Performing image registration and resizing on the $\mathrm{CCD}$ image with respect to the CMOS image are required prior to comparing the two sensors. In order to extract the data from the CCD image that corresponds to the CMOS image data, image registration between the two images and resizing allowed us to convert the CCD image $(1200 \mathrm{~V} \times 1600 \mathrm{H})$ to another image $(480 \mathrm{~V} \times 752 \mathrm{H})$. The correlation between the gray scale CCD registered image and the CMOS image is 82 percent, as computed by the MATLAB cross-correlation function (corr2). 


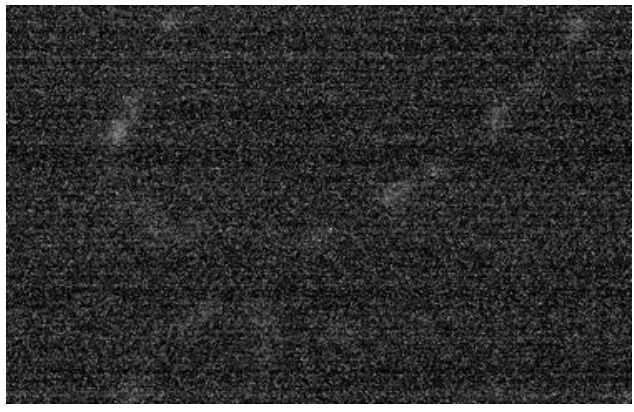

(a) CMOS Sensor - Before Noise Removal

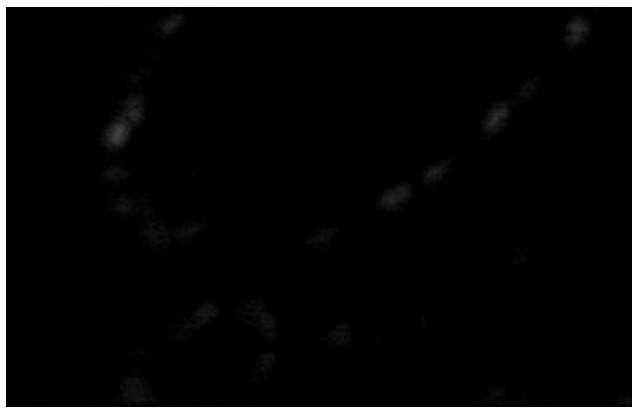

(c) CMOS Sensor - After FPN Removal and Median Filt. (3, 3)

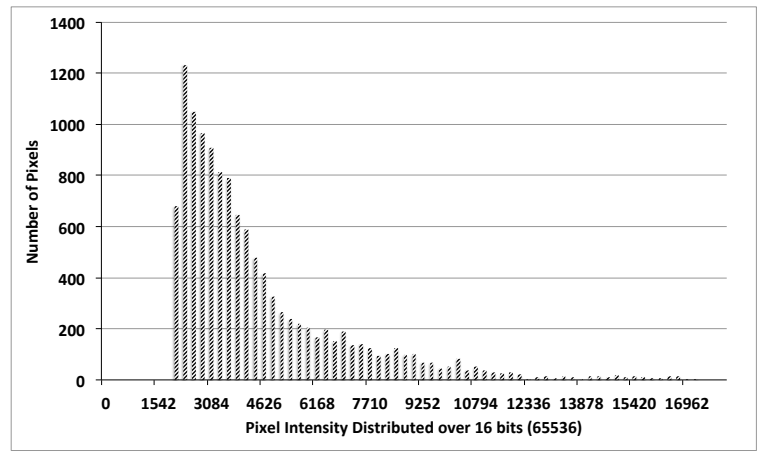

(e) CMOS Sensor - Histogram

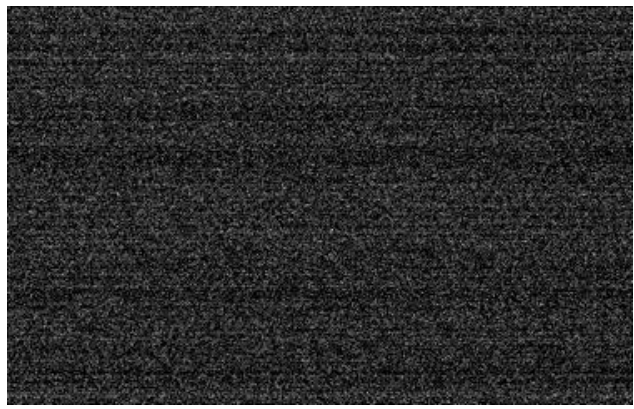

(b) CMOS Sensor - Master Dark Frame (MDF)

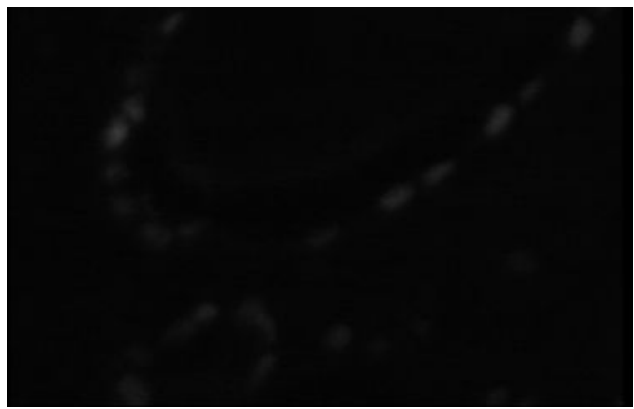

(d) CCD Sensor - After Median Filtering (3, 3)

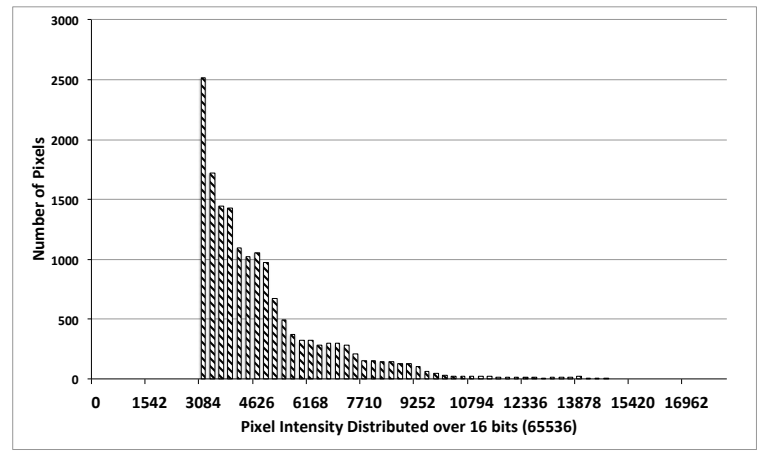

(f) CCD Sensor - Histogram

Figure 3: Examining Tissue Images Collected by the CMOS and CCD Cameras (Exposure=1s Analog Gain=8x)

\section{Intensity Distribution}

The histograms in Fig. 3e and Fig. 3f show the intensity distribution of the gray scale, noise reduced and registered (for $\mathrm{CCD}$ ) images over 16 bits. In this plot, $\mathrm{X}$ axis represents the intensity values distributed over 65536 values and the $Y$ axis represents the pixel counts corresponding to that specific intensity. For clarity purposes, the black pixels at 0 level intensity are left out of this graph and so the rest of the graph represent the biological information on the images. According to these figures, large majority of the pixels fall at low level intensities in both images since they are predominantly dark and the tissues show a large intensity distribution ranging from 3000 to 16000 . It is seen by the histograms that both images extract a similar intensity distribution.

\section{E. Auto Thresholding and Morphological Pattern Localization}

Thresholding is a process where individual pixels in an image with an intensity value greater than a defined threshold value are converted to 1 while all the other pixel values below this threshold are converted to 0 . To do that, the graythresh function of MATLAB is used to choose the threshold levels which computes the global threshold to minimize the intraclass variance of the bright and dark pixels as described by N. Otsu [10]. The resulting images as seen in Fig. 4 show that both cameras are capable of detecting the ER receptor expression at the nucleus that can be found in the breast tissue. The sum of the overall pixel values on the thresholded images provides the white pixel counts in an image and these values are normalized per pixel by dividing them to the total number of pixels. According to this calculation, the thresholded image collected by the CCD camera in Fig. 4b has 0.0448(\%4.448) normalized white pixel count while thresholded image of CMOS camera has 0.0338(\%3.38). Finally, when one to one matching among the pixels of each images are calculated, 84 percent of the white pixels in the CCD image found to be existing also in the CMOS image while 64 percent of the CMOS image 


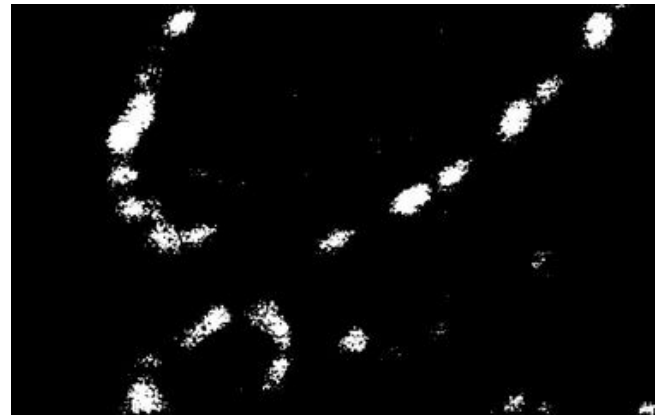

(a) CMOS Sensor - After Med. Filtering and Thresholding

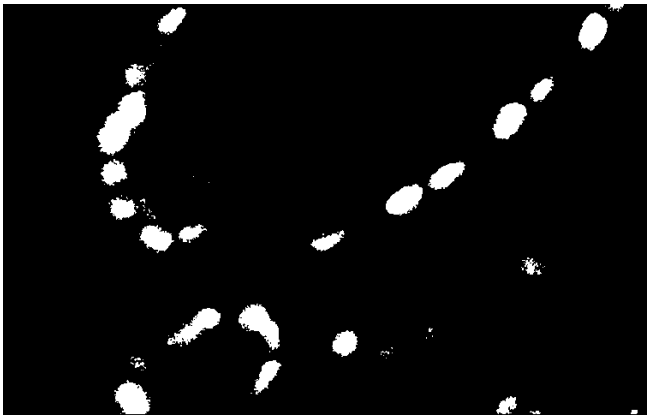

(b) CCD Sensor - After Med. Filtering and Thresholding

Figure 4: Detection of nuclear estrogen receptor (ER) expression with tissue samples obtained from breast cancer patients. ER is detected by indirect immunohistochemical reaction [11], [12] using monoclonal mouse anti-human anti-ER receptor antibody as primary antibody (clone 6F11, Leica Microsystems) and Alexa-Fluor 647 conjugated goat anti-mouse polyclonal IgG antibody (Invitrogen) as secondary anti-body.

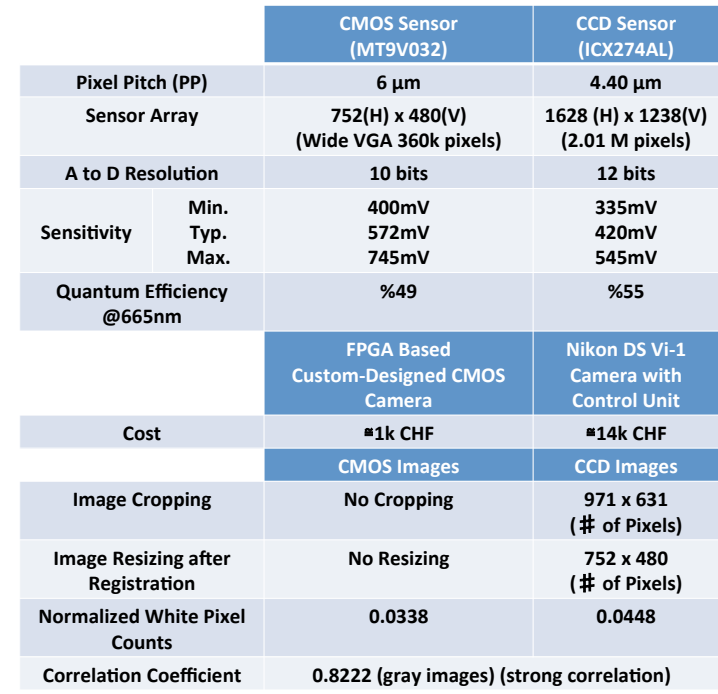

Figure 5: Comparison of the Performance Parameters and Performances of CMOS and CCD cameras

pixels in the CCD one. These variations were expected by the higher quantum efficiency of the CCD sensor plus its four times higher resolution of ADC (12 bits).

\section{CONCLUSION}

Fluorescence detection systems are of great importance for biological studies focused on the internal features of cells. The performances of imagers are the most important key parameter to succeed in such investigations. However, the cost and size of these systems plays a central role especially in future integration of imagers in lab-on-chip platforms. Shortly, by considering the one order of magnitude of cost difference between CMOS and CCD cameras plus the high integration capability of CMOS technology, in this paper we quantitatively compared a CCD and a mid-performance CMOS camera with respect to their performances for imaging on a biological tissue. We have shown that even a mid-performance CMOS camera can extract close enough information to that of a CCD camera when applied to get information on the spatial distribution of the fluorescent signals on an image. Therefore, this paper demonstrates that CMOS cameras may also be considered for quantitative investigations on cells or tissues when dealing with fluorescence microscopy.

\section{ACKNOWLEDGMENT}

The research work presented in this paper was funded by the NutriCHIP project with a grant from the Swiss NanoTera.ch initiative, evaluated by the Swiss National Science Foundation. It was also partially supported by the NanoSys project, the program ERC-2009-AdG-246810. Finally, the authors would like to thank to Ata Tuna Çiftlik from LMIS2 (Microsystems Laboratory 2), EPFL for their support in tissue sample preparation.

\section{REFERENCES}

[1] G. Mascetti, L. Vergani, A. Diaspro, S. Carrara, G. Radicchi and C. Nicolini, Effect of Fixatives on Calf Thymocytes Chromatin as Analyzed by 3D High-Resolution Fluorescence Microscopy, Cytometry, vol. 23(2), pp.110-119, 1996.

[2] G. Mascetti, S. Carrara and L. Vergani, Relationship between chromatin condensation compactness and dye uptake for in situ chromatin stained with DAPI, Cytometry, vol. 44(2), pp.110-119, 2001.

[3] C. Nicolini, S. Carrara and G. Mascetti, High order DNA structure as inferred by optical fluorimetry and scanning calorimetry, Mol. Biol. Rep., vol. 24, pp.235-346, 1997.

[4] J. Honghao, D. Sander, A. Haas and P.A. Abshire, Contact Imaging. Simulation and Experiment, IEEE Tran. on Circ. and Sys., vol. 54, no.8, pp.1698-1710, 2007.

[5] T. Tam, G. A. Jullien and O. Yadid-Pecht, A CMOS Contact Imager for Cell Detection in Bio-Sensing Applications, IEEE Int. Sym. on Circ. ans Sys. (ISCAS), pp.813 - 816, 2007.

[6] K. Murari, R. Etienne-Cummings, G. Cauwenberghs and N. Thakor, An integrated imaging microscope for untethered cortical imaging in freelymoving animals Conf. Proc. IEEE Eng Med. Biol. Soc., pp.5795-5798, 2010.

[7] K. Murari, et al. Design and characterization of a miniaturized epiilluminated microscope Proc. IEEE Eng. Med. Biol. Conf., pp.53695372, 2009.

[8] K. K. Ghosh et al. Miniaturized integration of a fluorescence microscope, Nature Methods, vol.8, pp.871-878, 2011.

[9] C. Favi, R. Beuchat, X. Jimenez and P. Ienne, From gates to multiprocessors learning systems hands-on with FPGA4U in a computer science programme, Proc. of the 2009 Workshop on Embed. Sys. Edu. (WESS), Grenoble, France, 2009.

[10] N. Otsu A threshold selection method from gray-level histograms, IEEE Trans. Systems, Man and Cybernetics (SMC), vol.9, no.1, pp.62-66, Jan.1979.

[11] B. Song, et al. Time-resolved lanthanide luminescence for lab-on-a-chip detection of biomarkers on cancerous tissues Proc. of the Nat. Academy of Sc. of the U.S.A, Vol. 107, Issue 31, pp. 13582-13587, August 2010.

[12] A.T. Ciftlik, et al. Fast Immunohistochemical BioMarker Detection Device for Cancer Tissue Slices, Proc. of 14th Int. Conf. on Miniaturized Systems for Chem. and Life Sc. (MicroTAS), pp. 699-70, Netherlands, Oct. 2010 